\title{
Determinação do Tecido Cicatricial do Miocárdio no Fenômeno de Fluxo Coronário Lento e a Relação entre a Quantidade de Tecido Cicatricial e o Nt-ProBNP
}

\author{
Determination of Myocardial Scar Tissue in Coronary Slow Flow Phenomenon and The Relationship Between \\ Amount of Scar Tissue and Nt-ProBNP
}

\author{
Mustafa Candemir, ${ }^{1,20}$ Asife Şahinarslan, ${ }^{2}$ Merve Yazol, ${ }^{3}$ Yusuf Ali Öner, ${ }^{4}$ Bülent Boyacl ${ }^{2}$ \\ Yozgat City Hospital - Department of Cardiology, ${ }^{1}$ Yozgat - Turquia \\ Gazi University - Faculty of Medicine - Department of Cardiology, ${ }^{2}$ Ankara - Turquia \\ Sanlıurfa Education and Research Hospital, Department of Radiology, ${ }^{3}$ Şanlıurfa - Turquia \\ Gazi University - Faculty of Medicine - Department of Radiology, ${ }^{4}$ Ankara - Turquia
}

\section{Resumo}

Fundamento: A fisiopatologia e o prognóstico não estão claramente determinados nos pacientes com fenômeno do fluxo coronário lento $(\mathrm{FCL})$. Esses pacientes apresentam várias condições clínicas, que variam desde quadro assintomático até internação hospitalar com morte cardíaca súbita.

Objetivos: Nosso objetivo foi avaliar os achados da ressonância magnética cardíaca (RMC) com o realce tardio pelo gadolínio (RTG), como um indicador de fibrose miocárdica. Também buscamos determinar a relação entre a presença de fibrose miocárdica e os níveis de NT-proBNP em pacientes com FCL na artéria coronária descendente anterior esquerda (DAE).

Métodos: Ao todo, 35 pacientes, entre 31 e 75 anos de idade, foram incluídos. Os pacientes estudados ( $n=19$ ) apresentaram artérias coronárias epicárdicas normais na angiografia, mas tinham FCL na DAE. O grupo controle de pacientes $(n=16)$ apresentou artérias coronárias epicárdicas normais e níveis de escore TIMI normais na angiografia. Em ambos os grupos, os pacientes foram examinados com RMC para a detecção de presença de fibrose miocárdica. Além disso, níveis plasmáticos de NT-proBNP foram medidos. Valores de $p<0,05$ foram considerados significativos.

Resultados: A taxa de fibrose miocárdica foi significativamente maior na RMC para os pacientes Com FCL ( $p=0.018)$. Uma quantidade variável de tecido cicatricial foi detectada no ápice ventricular esquerdo em 7 pacientes e nas regiões inferior e inferolateral em 3 pacientes. Não foram observadas diferenças nos níveis de NT-proBNP nos pacientes com FCL. Entretanto, os níveis de NT-proBNP foram maiores nos pacientes com FCL, que apresentaram fibrose miocárdica na $\mathrm{RMC}(\mathrm{p}=0.022)$.

Conclusões: Em suma, o RTG na RMC mostrou que a cicatriz miocárdica isquêmica pode estar presente nos pacientes com FCL. Esses resultados indicam que o FCL pode nem sempre ser inofensivo. (Arq Bras Cardiol. 2020; 114(3):540-551)

Palavras-chave: Insuficiência Cardiaca; Reserva Fracionada de Fluxo Miocárdico; Cicatriz Hipertrófica; Prognóstico; Peptídeo Natriurético Tipo C; Fibrose Endomiocárdica; Espectroscopia de Ressonância Magnética/métodos.

\section{Abstract}

Background: Pathophysiology and prognosis are not clearly determined in patients with the coronary slow flow phenomenon (CSFP). These patients present with various clinical conditions ranging from being asymptomatic to being admitted with sudden cardiac death.

Objectives: We aimed at assessing the findings of late gadolinium enhancement (LGE) in cardiac magnetic resonance imaging (CMR) as an indicator of myocardial fibrosis. We also aimed at determining the relationship between the presence of myocardial fibrosis and NT-proBNP levels in patients with CSFP in the left anterior descending coronary artery (LAD).

Methods: A total of 35 patients were enrolled within an age range of 31-75. The study patients $(n=19)$ had normal epicardial coronary arteries at angiography, but they presented with CSFP in the LAD. The control group patients $(n=16)$ had normal epicardial coronary arteries and TIMI scores at normal levels in angiography. In both groups, the patients were examined with CMR for the presence of myocardial fibrosis. In addition, plasma NT-proBNP levels were measured. A p-value $<0.05$ was considered significant.

Results: The rate of myocardial fibrosis was significantly higher in CMR in the patients with CSFP $(p=0.018)$. A variable amount of myocardial scar tissue was detected at the left ventricular apex in 7 patients and at the inferior and inferolateral regions in 3 patients. There was no difference in the level of NT-proBNP in patients with CSFP. However, the NT-proBNP levels were higher in patients with CSFP, who had scar tissue in CMR ( $p=0.022$ ). Conclusions: In conclusion, LGE in CMR showed that ischemic myocardial scarring may exist in patients with CSFP. These results indicate that CSFP may not always be innocent. (Arq Bras Cardiol. 2020; 114(3):540-551)

Keywords: Heart Failure; Fractional Flow Reserve, Myocardial; Cicatrix, Hypertrophic; Prognosis; Natriuretic-Peptide, C-Type; Endomyocardial Fibrosis, Magnetic Resonance Spectroscopy.

Full texts in English - http://www.arquivosonline.com.br

Correspondência: Mustafa Candemir •

Yozgat City Hospital, Department of Cardiology, Yozgat, 66100, Turkey

E- mail: mstfcndmr@hotmail.com

Artigo recebido em 15/08/2019, revisado em 27/04/2019, aceito em 05/06/2019

DOI: https://doi.org/10.36660/abc.2018149 


\section{Introdução}

Há pouca informação na literatura em relação ao prognóstico do fenômeno do fluxo coronário lento ( $\mathrm{FCL}$ ). Dados preexistentes indicam que a isquemia miocárdica relacionada ao fluxo lento pode causar angina e o prognóstico é pior nesses pacientes. ${ }^{1}$ Também relatou-se uma associação entre infarto agudo do miocárdio, ${ }^{2}$ morte cardíaca súbita e arritmia ventricular maligna e o $\mathrm{FCL}^{3}{ }^{\mathrm{A}}$ ocorrência de episódios recorrentes de dor torácica ou dor torácica em repouso, bem como elevadas taxas de internações de emergência e hospitalizações, foram relatadas. ${ }^{4,5}$ Desse modo, esse fenômeno não é tão inofensivo como parece mas, ao contrário, possui potencial para causar séria deterioração na qualidade de vida. Não se sabe ao certo atualmente se há lesões orgânicas nesses pacientes, devido à ausência de investigações e resultados mais aprofundados.

Os níveis de N-terminal do pró-hormônio do peptídeo natriurético do tipo B (NT-proBNP) aumentaram após o exercício nos pacientes com fluxo coronário lento. ${ }^{6}$ Há uma correlação entre a isquemia ou o tamanho do infarto na ressonância magnética (RMI), e essa correlação também pode ser observada nos níveis de NT-proBNP nos pacientes com síndrome coronariana aguda. ${ }^{7}$ Com os avanços na ressonância magnética cardíaca (RMC), a isquemia microvascular e a fibrose cardíaca podem ser demonstradas através dessa técnica. ${ }^{8,9}$ A relação entre a extensão da fibrose e os níveis de NT-proBNP nesses pacientes já foi revelada em vários exames de RMI realizados em pacientes com síndrome aguda coronariana. ${ }^{10,11}$ Entretanto, não há estudos na literatura que tenham avaliado pacientes com FCL para detecção da presença de fibrose no tecido do miocárdio, de acordo com os resultados do realce tardio pelo gadolínio (RTG) na RMC. O objetivo deste estudo foi investigar a presença de fibrose miocárdica em pacientes com fluxo lento na artéria coronária descendente anterior esquerda por meio da técnica do realce tardio pelo gadolínio na RMC, bem como avaliar a relação entre a fibrose miocárdica e os níveis de NT-proBNP.

\section{Materiais e Métodos}

\section{População do Estudo}

Dentre os pacientes que foram admitidos no nosso departamento entre janeiro de 2015 e agosto de 2016, e submetidos à angiografia coronária devido a dores torácicas, 19 pacientes com o fenômeno do fluxo coronário lento na DAE foram incluídos neste estudo de coorte prospectivo. O grupo controle incluiu dezesseis pacientes cujas artérias epicárdicas estavam completamente normais com fluxo coronário normal.

Este estudo foi aprovado pelo Comitê de Ética do Hospital Universitário de Gazi e conduzido em conformidade com os princípios da declaração de Helsinki.

\section{Critérios de Exclusão}

Os seguintes pacientes foram excluídos do estudo: pacientes com ectasia de artéria coronariana ou lesões ateroscleróticas nas artérias coronárias esquerda e descendente anterior esquerda; pacientes submetidos à intervenção coronária percutânea; pacientes com intervenção da artéria coronária agendada; pacientes com estenose $>50 \%$ em qualquer artéria coronária; pacientes com história prévia de IM; pacientes com função sistólica do ventrículo esquerdo $<50 \%$; pacientes com claustrofobia, insuficiência cardíaca ou disfunção valvar, extra-sístoles ventriculares ou anormalidades da condução atrioventricular e bloqueio de ramo ou fibrilação atrial; pacientes com teste de esforço positivo; pacientes com cardiomiopatias restritiva, hipertrófica ou dilatada; pacientes com doença sistêmica conhecida (hipertireoidismo, hipotireoidismo, malignidade, doença autoimune, infecção ou quaisquer distúrbios pulmonares, hepáticos, renais, hematológicos); pacientes com história prévia de miocardite, com TFG $<80 \mathrm{ml} / \mathrm{min}$, e pacientes que se recusaram a participar do estudo.

\section{Dados dos Pacientes}

Foram medidos a altura, o peso e o índice de massa corporal (IMC) dos pacientes do estudo. A idade, o gênero, os fatores de risco cardiovascular (hipertensão, diabetes, dislipidemia, tabagismo e histórico familiar), características demográficas e comorbidades foram registrados. Foi obtido eletrocardiograma (ECG) de todos os pacientes e todos eles apresentaram ritmo sinusal. Todos os pacientes do estudo foram examinados na posição decúbito lateral direita, utilizando um sistema de ultrassom Vivid 7-Pro (GE Vingmed, Horten, Noruega), equipado com um transdutor de 2,5 MHz, através do registro simultâneo pelo ECG de 1 derivação. Medições em modo-M e Doppler foram realizadas em conformidade com as recomendações da Associação Americana de Ecocardiografia. ${ }^{12}$

O teste de esforço foi realizado em média 3 dias antes da angiografia em todos os pacientes (GE medical system, Milwaukee, EUA), de acordo com o protocolo padrão de Bruce, sendo o ECG convencional, além das medições da pressão arterial e da frequência cardíaca realizadas em períodos de tempo pré-definidos, conforme dizetrizes relevantes. ${ }^{13}$

Foram coletadas amostras de sangue para dosagem de NT-proBNP, através da bainha vascular, imediatamente antes da sua retirada. Após a coleta, elas foram centrifugadas por 10 minutos a 4500 RPM e armazenadas a -20 ㄷ C até a realização dos testes sorológicos. No dia da análise, após as amostras atingirem a temperatura ambiente, um imunoensaio eletroquimioluminescente foi realizado com o Analisador Cobas e 411 (Roche Diagnostics GmbH, Mannheim, Alemanha). Os resultados foram apresentados em picogramas por $\mathrm{ml}(\mathrm{pg} / \mathrm{ml})$. O coeficiente de variação do NT-proBNP, através desse método, foi inferior a 5\%.

\section{Angiografia Coronária e Contagem de Quadros TIMI}

A angiografia coronária foi realizada utilizando-se a técnica padrão de Judkins com acesso femoral e a 30 imagens por segundo, através do sistema de angiografia Infinix (Toshiba Corporation, Tochigi, Japão). A iopromida (Ultravist-370; Bayer Pharma AG, Berlim, Alemanha) foi utilizada como agente de contraste durante a angiografia coronária. Uma média de 6 a $8 \mathrm{ml}$ do agente de contraste foi injetada 
manualmente para cada exposição. As artérias coronárias foram visualizadas em projeção oblíqua esquerda e direita no ângulo cranial-caudal apropriado. A velocidade do fluxo na DAE foi avaliada nas projeções oblíquas anterior direita e esquerda, frequentemente com ângulo caudal. As imagens foram avaliadas por dois especialistas clínicos cegos em relação às condições clínicas dos pacientes.

A avaliação quantitativa do fluxo coronário foi realizada de acordo com o estudo TIMI-4, através da contagem de quadros angiográficos, a partir do momento da administração do agente de contraste, até a sua chegada a um determinado ponto distal. ${ }^{14}$ A metodologia da contagem de quadros foi padronizada para cada vaso epicárdico. A contagem de quadros TIMI começou com o primeiro quadro no qual o contraste preencheu completamente a artéria. O preenchimento completo da artéria foi determinado pelo cumprimento dos três critérios a seguir: (1) Uma coluna de contraste total ou quase totalmente concentrado deve se estender ao longo de toda a origem da artéria coronária; (2) O contraste toca ambas as bordas de origem da artéria coronária; e (3) Deve haver um um fluxo anterógrado de contraste. O último quadro a ser contado foi aquele no qual o contraste preencheu inicialmente o ramo distal da artéria alvo. A opacificação completa do segmento distal não foi necessária.

O escore TIMI para a DAE foi 1,7 vez mais elevado do que a contagem média da ACD e da ACx. Consequentemente, a contagem do escore TIMI para a DAE foi dividida por 1,7 para calcular a contagem quadro a quadro TIMI corrigida (CTFC).

No nosso estudo, o fluxo coronário para a DAE foi considerado normal se a contagem do escore TIMI $<23$ e foi definida como lenta se a contagem do escore TIMI $\geq 23$. $^{15,16}$

\section{Técnica da Ressonância Magnética}

A RMC foi realizada após uma mediana de 8 dias (0-21 dias) após a angiografia coronária. Sequências padronizadas de estudos sobre perfusão pela RMC foram utilizadas em todos os pacientes. A veia antecubital esquerda foi usada para injeção do contraste intravenoso. Os dados de RM dos pacientes foram adquiridos utilizando um sistema de IRM 3T (Siemens MAGNETOM $®$ Verio, Erlangen, Alemanha) equipado com gradiente de potência de $45 \mathrm{mT} / \mathrm{m}$. Uma bobina de 6 canais foi colocada na parede torácica frontal enquanto o paciente estava deitado em decúbito dorsal com as almofadas de ECG posicionadas corretamente. Foram obtidas aquisições multiplanares com uma sequência turbo-FLASH de inversão-recuperação sensível à fase (PSIR) em múltiplas apneias expiratórias. As imagens padrão do coração de eixo longo, 2 câmaras, 4 câmaras e eixo curto foram obtidas alinhando a válvula mitral e o ápice. Os parâmetros de imagem foram: TR (tempo de repetição): 800; TE (tempo de eco) 6,66 ms; espessura das fatias: $8 \mathrm{~mm}$; matriz: 128x256; FOV (campo de visão): 400 mm. Imagens ponderadas em T1 e T2 acompanhadas de sequência de pulsos "inversão-recuperação" para atenuação de sinais de sangue (sangue escuro) e de sequência-SPIN ECO foram adquiridas para avaliar a morfologia do miocárdio (TR/TE/ espessura/ matriz/ FOV: 698/6,6/ 8 mm/ 128x256, 360 $\mathrm{mm}$ ). Foram adquiridas imagens de perfusão miocárdica dinâmica de primeira passagem uilizando-se a sequência de pulso SR Turbo FLASH (Tfl), após administração de uma dose de 0,025 mmol/kg de Gd-DTPA intravenoso (Magnevist; Bayer Healthcare, Wayne NJ, EUA). Em intervalos de oito minutos de repouso, uma série de imagens de "gradienteeco" (cine-RM) no plano de eixo curto foram adquiridas para avaliação funcional dos ventrículos ao longo do ciclo cardíaco, utilizando-se a apneia (TR/TE/ espessura/ matriz/ FOV: 40.24/ TE/ $8 \mathrm{~mm} / 128 \times 256 / 360 \mathrm{~mm}$ ). Imagens ponderadas em T1, de eixo curto e 4 câmaras, foram obtidas na sequência PSIR, aproximadamente 8 minutos após a administração do contraste (TR/TE/ espessura/ matriz/ FOV: 756/ TE: 1,15/ $6 \mathrm{~mm} / 128 \times 256 / 360 \mathrm{~mm}$ ). O tempo total de duração do exame foi de 35 minutos em média.

Os custos da RMC e da dosagem plasmática do NTproBNP foram cobertos pela Unidade de Projetos de Pesquisa Científica da Universidade de Gazi.

\section{Análise de Imagem por Ressonância Magnética}

Todas as imagens da RMC foram transferidas para a estação de trabalho para análise (Siemens, Leonardo multimodality workplace, Siemens Healthcare). Todas as avaliações foram realizadas visualmente. Os exames de RMC foram avaliados retrospectivamente por um radiologista com mais de 15 anos de experiência em imagem cardíaca, de forma cega aos resultados da ecocardiografia e da angiografia coronária. Ao se observar quaisquer defeitos de perfusão ou realce tardio durante essas avaliações, os mesmos eram registrados de forma precisa. O realce do contraste nas sequências de perfusão foi definido como o cumprimento de todas as 5 fases após a obtenção da intensidade de sinal mais alta no ventrículo esquerdo. Os resultados da RMC foram então comparados com os resultados da ecocardiografia e da angiografia coronária dos pacientes.

\section{Análise Estatística}

Utilizou-se o programa estatístico SPSS (versão 21.0, SPSS Inc., Chicago, Illinois, EUA) para a análise de dados do estudo. Foram utilizados métodos visuais (histogramas, gráficos de probabilidade) e analíticos (teste KolmogorovSmirnov) para determinar a normalidade das variáveis. Nas análises descritivas, as variáveis distribuídas normalmente foram apresentadas como valor e desvio padrão, enquanto que as não normais foram apresentadas como mediana (intervalo interquartil). As variáveis categóricas foram apresentadas como percentuais. Amostras independentes do Teste-t e Mann-Whitney $U$ foram usadas para comparar as variáveis numéricas. A análise do qui-quadrado de Pearson foi utilizada para comparar os dados categóricos, mas o teste exato de Fisher foi realizado quando dois dos valores esperados ficavam abaixo de 5 ou um dos valores esperados fosse menor que 2 . Valores de $p<0,05$ foram considerados estatisticamente significativos.

\section{Resultados}

Ao todo, 35 pacientes foram incluídos no estudo. Os pacientes foram divididos em 2 grupos: o grupo de pacientes e o grupo controle. Dezenove pacientes foram incluídos no 
grupo com fluxo lento na DAE, e 16 pacientes com fluxo coronário normal foram incluídos no grupo controle (Figura 1). Os pacientes do grupo controle foram comparados, quanto aos fatores de risco, aos indivíduos dos grupos de pacientes. A idade média dos pacientes era de 50,3 \pm 10,7, e 6 dos 35 pacientes (17\%) eram mulheres. Onze pacientes (31\%) eram diabéticos, 9 pacientes (25\%) eram hipertensos, 5 pacientes (14\%) eram dislipidêmicos, 18 pacientes (51\%) eram tabagistas e 8 pacientes (22\%) tinham histórico familiar positivo (Tabela 1).

A principal queixa de todos os pacientes era dor torácica. O ECG de todos os pacientes mostrou ritmo sinusal. A frequência cardíaca variou entre 64 e 92 bpm. A frequência cardíaca média foi $74 \mathrm{bpm}$. Além disso, não houve sinal de isquemia, hipertrofia ou arritmia ao ECG. A fração de ejeção do ventrículo esquerdo e outros achados ecocardiográficos dos pacientes estavam normais. Adicionalmente, os testes de esforço de todos os pacientes foram negativos. A troponina de alta sensibilidade foi medida antes e depois da angiografia coronária em todos os pacientes. Todos os valores ficaram abaixo do valor limite e não houve aumento nos valores de troponina após a angiografia.

O tempo de intervalo entre os exames de RMC e a angiografia coronária dos pacientes não deveria ser superior a 21 dias.

Quando os pacientes com fluxo lento foram comparados com o grupo controle, nenhuma diferença significativa foi observada nos valores de NT-proBNP $(p=0,247)$. Os resultados positivos da RMC foram significativamente mais comuns nos pacientes com fluxo lento $(p=0,001)$ (Table 1 ). Tecido cicatricial foi observado em níveis variáveis no ápice cardíaco de 7 pacientes (Figuras 2 e 5) e nas regiões inferior e inferolateral em 3 pacientes (Figures 3, 4, 6, 7 and 8). Nenhum tecido cicatricial foi observado em 9 pacientes (Figure 9).

As características demográficas e os graus de fluxo TIMI não foram diferentes no grupo positivo à RCM quando comparado com o grupo negativo à RMI. (Tabela 2). Os níveis de NT-proBNP foram estatisticamente significativos nos pacientes com fluxo lento e tecido cicatricial à RMC $(\mathrm{p}=0,022)$ (Tabela 2).

Todos os indivíduos completaram o teste ergométrico

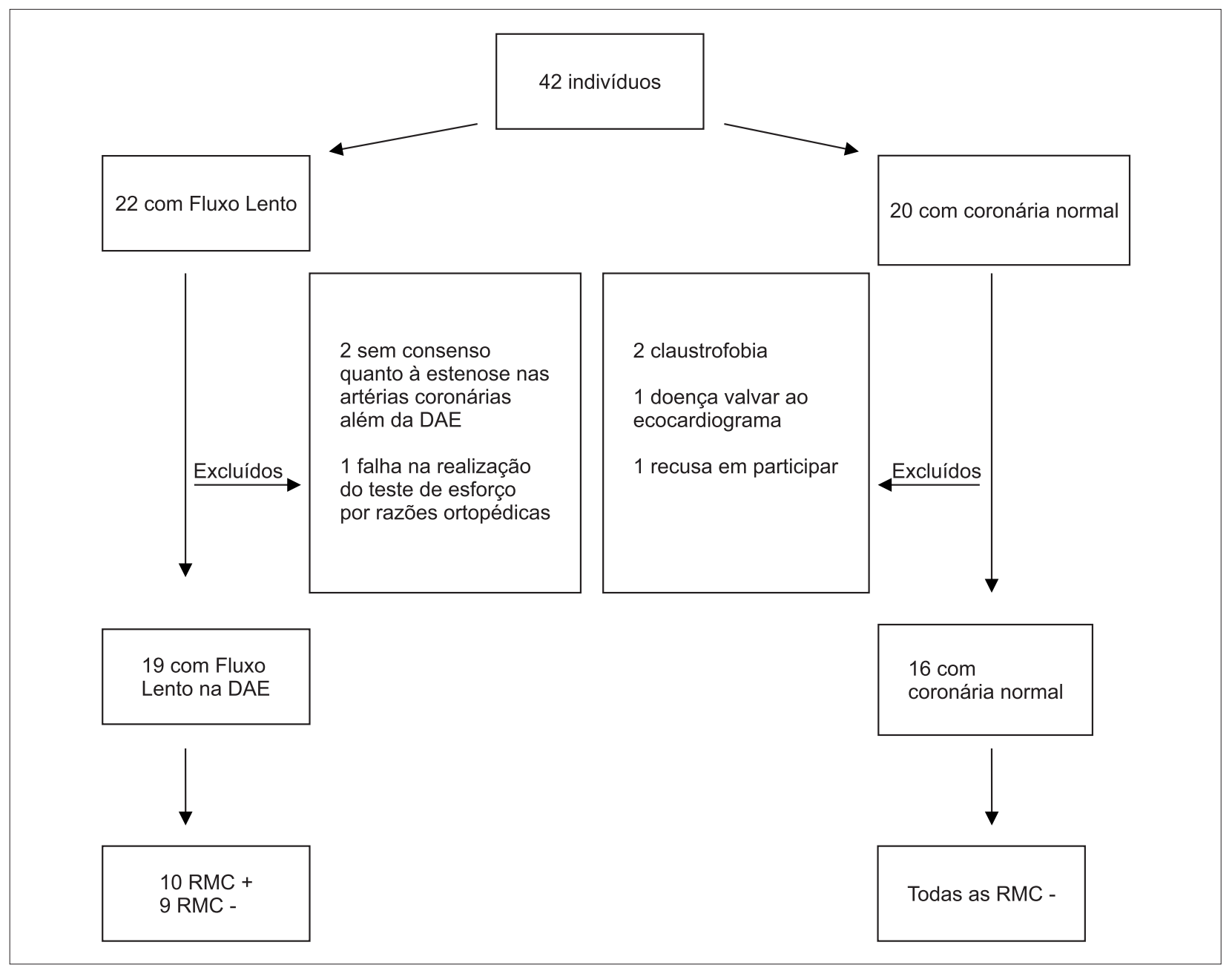

Figura 1 - Seleção de pacientes. RMC: imagem por ressonância magnética cardíaca. 
Candemir et al.

Fluxo Lento e Imagem por Ressonância Magnética

\section{Artigo Original}

\section{Tabela 1 - Comparação das características clínicas de ambos os grupos}

\begin{tabular}{|c|c|c|c|c|}
\hline Parâmetros & $\begin{array}{c}\text { Total } \\
(\mathrm{N}=35)\end{array}$ & $\begin{array}{l}\text { Fluxo Lento } \\
\qquad(N=19)\end{array}$ & $\begin{array}{l}\text { Controle } \\
(\mathrm{N}=16)\end{array}$ & Valor de $p$ \\
\hline Idade, média (DP), anos & $50,3 \pm 10,7$ & $51,3 \pm 8,2$ & $49,44 \pm 12,8$ & 0,62 \\
\hline Sexo (Masculino), n (\%) & $29(82)$ & $15(78,9)$ & $14(87,5)$ & 0,50 \\
\hline Hipertensão, n (\%) & $9(25)$ & $6(31,6)$ & $3(18,8)$ & 0,38 \\
\hline Diabetes mellitus, n (\%) & $11(31)$ & $6(31,6)$ & $5(31,3)$ & 0,98 \\
\hline Tabagista, n (\%) & $18(51)$ & $9(47,4)$ & $9(56,3)$ & 0,60 \\
\hline Histórico familiar, n (\%) & $8(22)$ & $4(21,1)$ & $4(25)$ & 0,78 \\
\hline Dislipidemia, n (\%) & $5(14)$ & $3(15,8)$ & $2(12,5)$ & 0,78 \\
\hline IMC, média (DP) (kg/m²) & $27,7 \pm 2,3$ & $28,1 \pm 2,5$ & $27,3 \pm 2$ & 0,39 \\
\hline NT-proBNP (pg/ml) & $29,5(17,7-66,2)$ & $47,8(22,6-121,5)$ & $26,0(10,9-58,1)$ & 0,246 \\
\hline cTFC (quadro/segundo) & $34,6 \pm 16,2$ & $28,0 \pm 8,6$ & $13,1 \pm 1,2$ & $<0,001$ \\
\hline METs, $\mathrm{mL} / \mathrm{kg} / \mathrm{dk}$ & $10,38 \pm 1,91$ & $9,74 \pm 2,05$ & $11,15 \pm 1,43$ & 0,027 \\
\hline Resultados positivos da IMR n (\%) & $10(28)$ & $10(52,6)$ & $0(0)$ & 0,001 \\
\hline
\end{tabular}

IMC: índice de massa corporal; cTFC: TIMI corrigido; METs: equivalentes metabólicos; RMI: ressonância magnética por imagem.

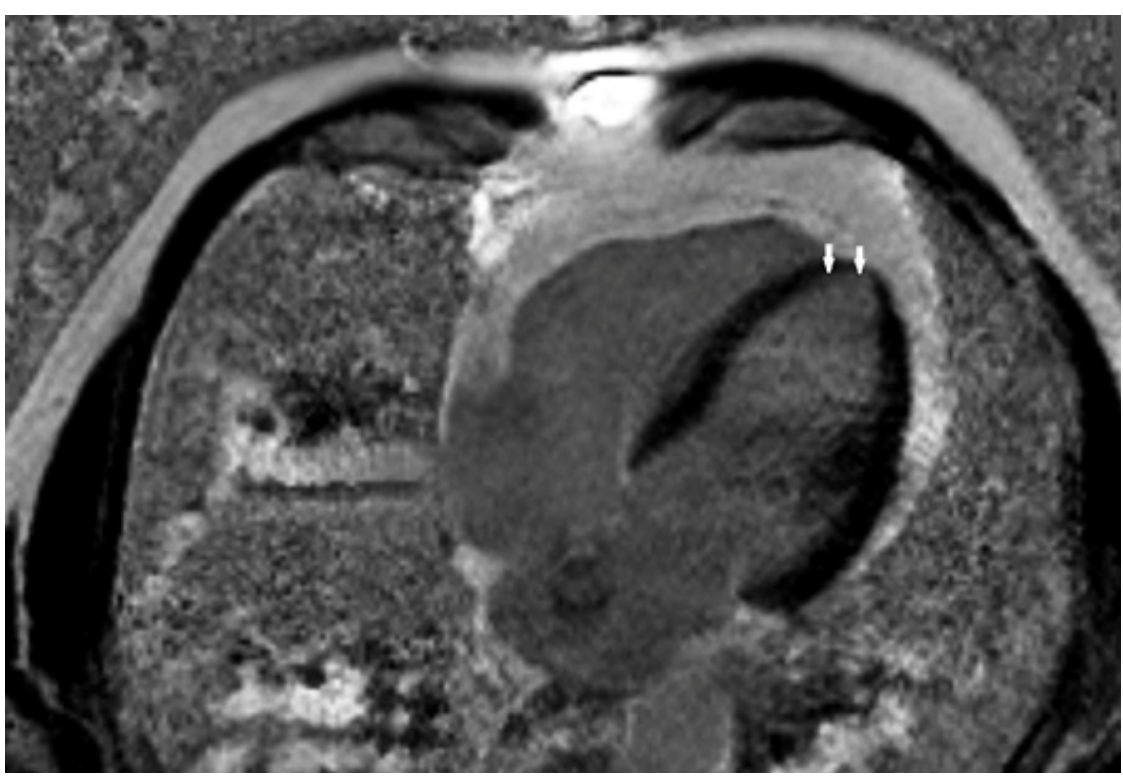

Figura 2 - Imagem no plano 4 câmaras, na sequência inversão-recuperação sensível à fase (PSIR) com realce tardio, revelando realce tardio subendocárdico circunferencial na região apical (setas) do ventrículo esquerdo. 


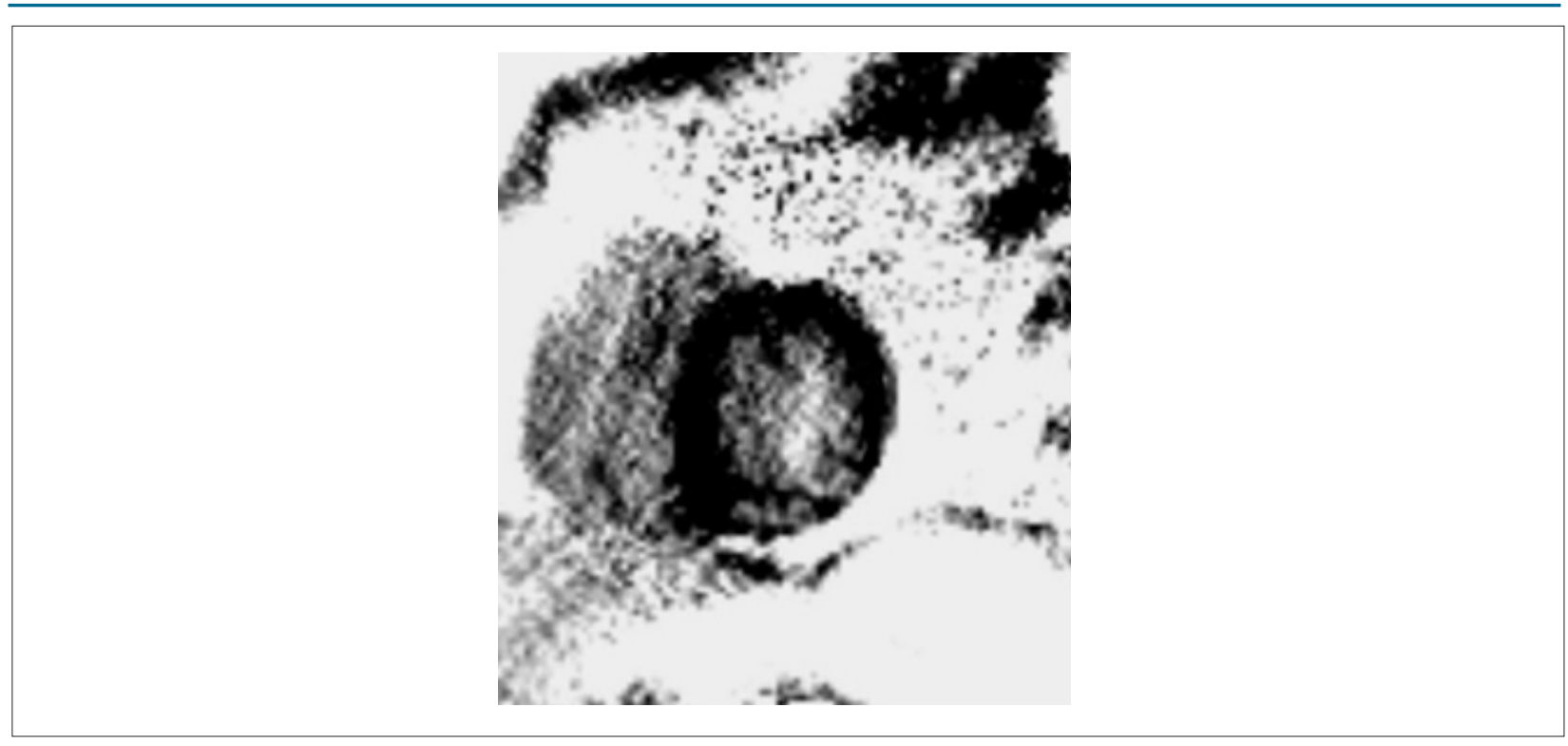

Figura 3 - Imagem de RMC na sequência PSIR com contraste tardio no eixo curto, revelando áreas e focos de realce tardio em regiões subendocárdicas (transmurais) e subepicárdicas, sobretudo nas paredes do ventrículo esquerdo inferior e inferolateral.

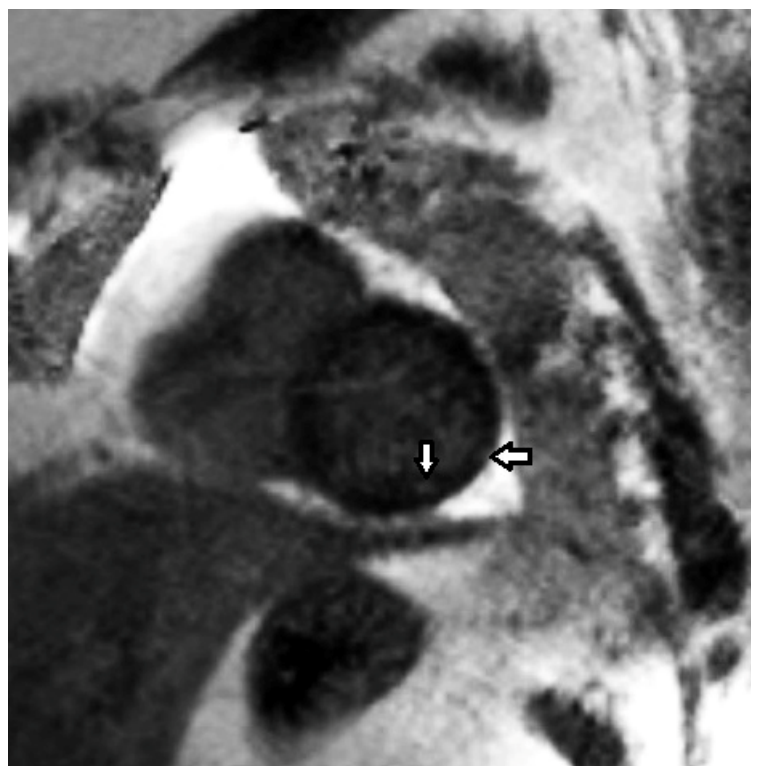

Figura 4 - Imagem de RMC na sequência PSIR com contraste tardio no eixo curto, revelando focos de realce tardio em regiões subendocárdicas e subepicárdicas localizadas nas paredes inferior e inferolateral do VE (setas). Essas áreas hiperintensas refletem tecidos cicatriciais fibróticos que se espalham para o território da DAE. 


\section{Artigo Original}

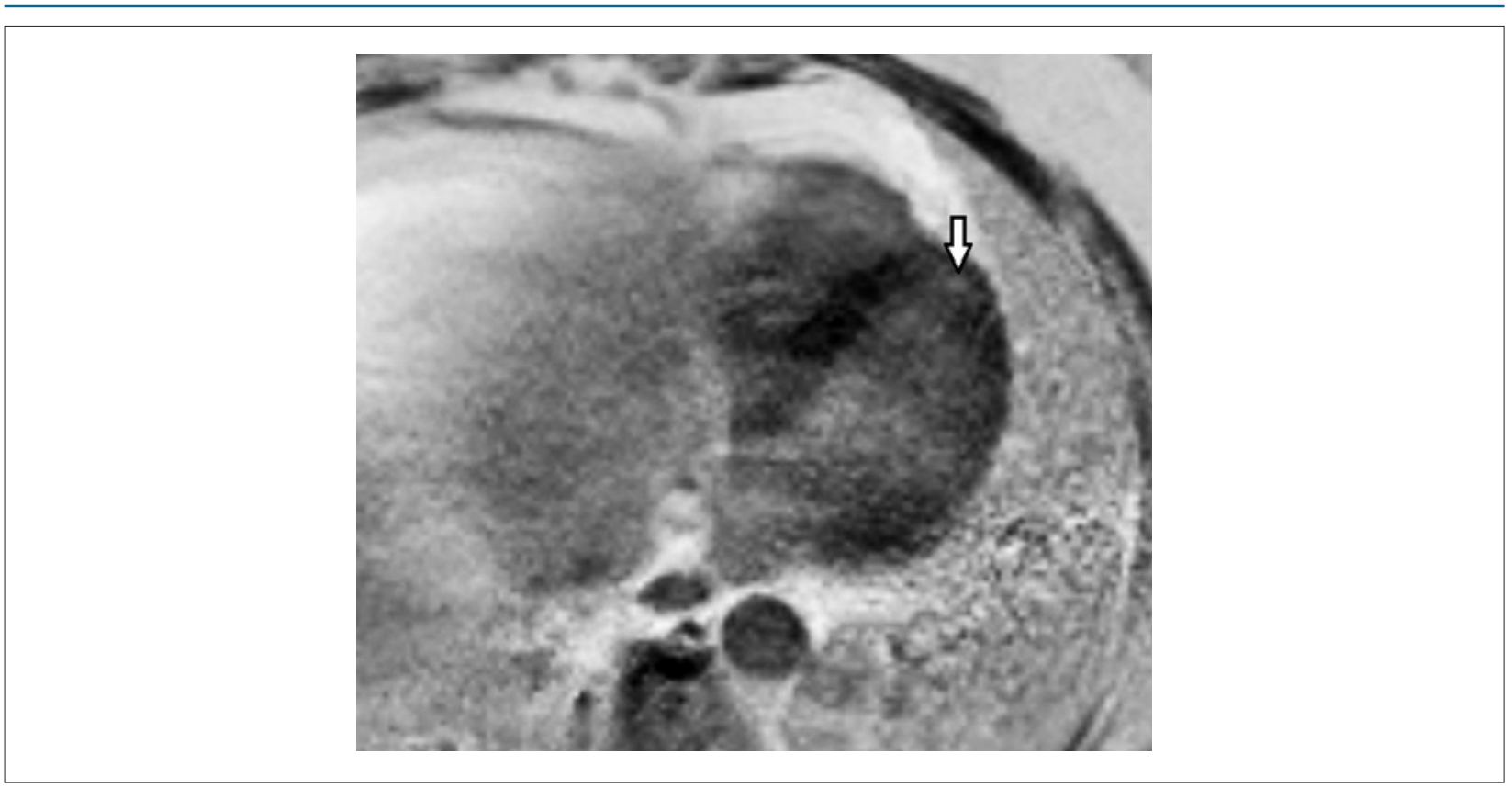

Figura 5 - Lesão focal em imagem ponderada em T1 no plano 4 câmaras, na sequência PSIR, com realce tardio pelo gadolínio. Lesão focal localizada na região apical do ventrículo esquerdo, revelando realce tardio subendocárdico-miocárdico compativel com fibrose.

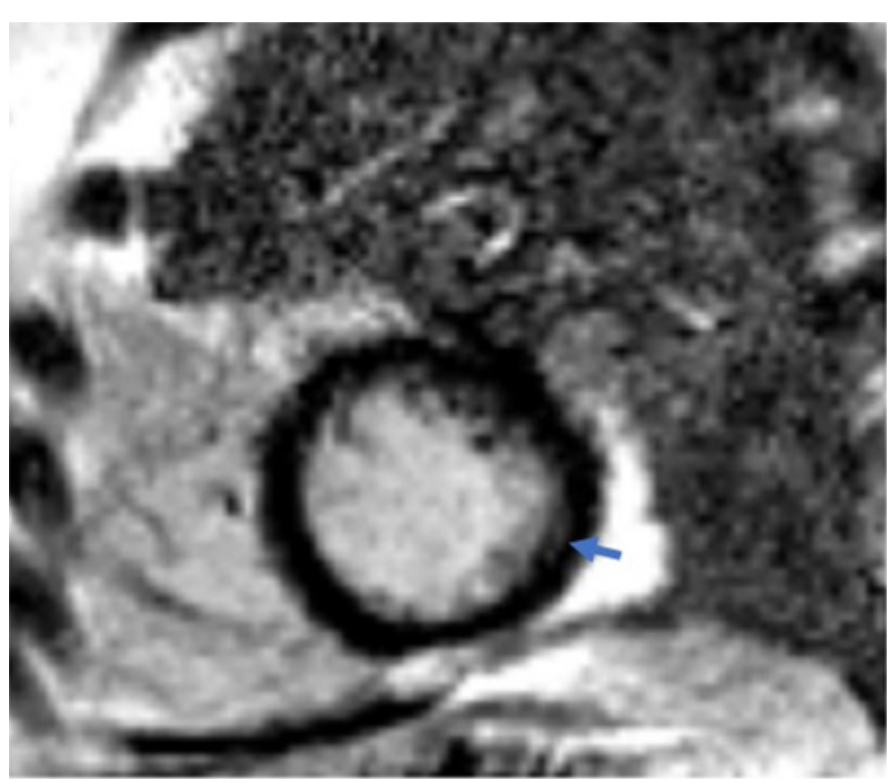

Figura 6 - Imagem de sequência PSIR, com realce tardio, no eixo curto, representando áreas de realce subendocárdicas, sobretudo na parede ventricular esquerda inferolateral. Tecido cicatrial abrangendo quase $25-50 \%$ da espessura da parede. 


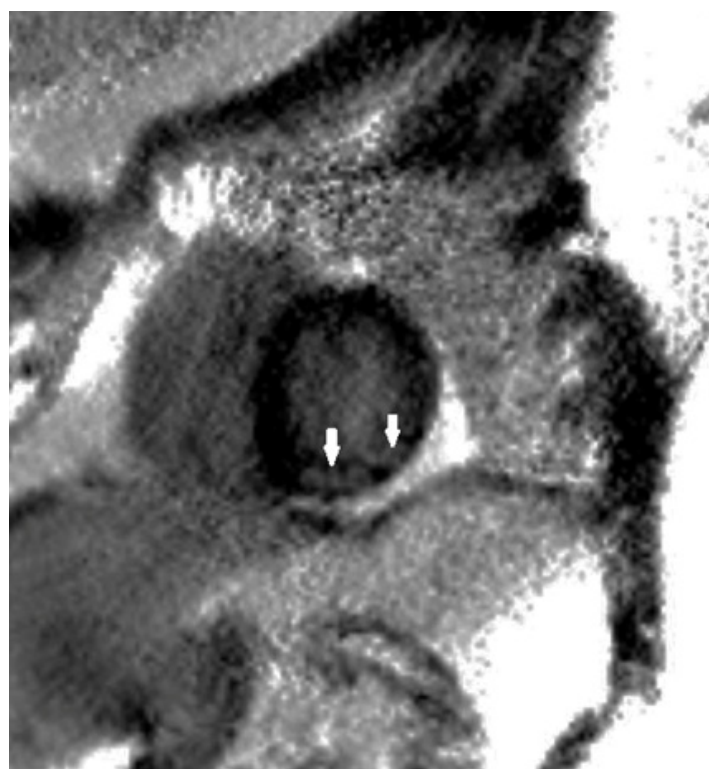

Figura 7 - Imagem de RMC com sequência PSIR pela técnica do contraste tardio no eixo curto, revelando áreas de realce focal nas regiões subepicárdica (transmural) e subendocárdica, sobretudo nas paredes inferior e inferolateral do VE (setas), indicando tecidos cicatriciais na distribuição da DAE.

Tabela 2 - Características clínicas dos pacientes com fluxo lento

\begin{tabular}{|c|c|c|c|}
\hline \multirow{2}{*}{ Parâmetros } & \multicolumn{3}{|c|}{ Fluxo Lento $(\mathrm{N}=19)$} \\
\hline & RMI cardíaca $(+)(\mathrm{N}=10)$ & RMI cardíaca (-) (N= 9) & Valor de $p$ \\
\hline Idade, média (DP), anos & $54,1 \pm 9,6$ & $49,4 \pm 7,1$ & 0,29 \\
\hline Sexo (Masculino), n (\%) & $6(60)$ & $9(100)$ & 0,08 \\
\hline Hipertensão, n (\%) & $4(40)$ & $2(22,2)$ & 0,62 \\
\hline Diabetes mellitus, n (\%) & $3(30)$ & $3(33,3)$ & 1,0 \\
\hline Tabagista, n (\%) & $6(60)$ & $3(33,3)$ & 0,37 \\
\hline Histórico familiar, n (\%) & $1(10)$ & $3(33,3)$ & 0,30 \\
\hline Dislipidemia, n (\%) & $3(30)$ & $0(0)$ & 0,21 \\
\hline IMC, média (DP) $\left(\mathrm{kg} / \mathrm{m}^{2}\right)$ & $28,2 \pm 3,0$ & $28,0 \pm 2,4$ & 0,81 \\
\hline NT-proBNP (pg/ml) & 147,10 & $28,0(21,5-56,2)$ & 0,03 \\
\hline cTFC (quadro/segundo) & $(41,57-734,57)$ & $26,4(22,9-35,0)$ & 0,67 \\
\hline METs, mL/kg/dk & $24,1(23,8-28,9)$ & $10,26 \pm 1,92$ & 0,304 \\
\hline
\end{tabular}

IMC: índice de massa corporal; cTFC: TIMI corrigido; METs: equivalentes metabólicos; RMI: ressonância magnética por imagem.

Tabela 3 - Resultados dos testes de esforço por grupos

\begin{tabular}{lccccc}
\hline Parâmetro & $\begin{array}{c}\text { Controle } \\
(\mathrm{N}=16)(1)\end{array}$ & $\begin{array}{c}\mathrm{RM} \text { Cardíaca } \\
(+)(\mathrm{N}=10)(2)\end{array}$ & $\begin{array}{c}\text { RM Cardíaca } \\
(-)(\mathrm{N}=9)(3)\end{array}$ & Valor de $p(1-2)$ & Valor de $p(1-3)$ \\
\hline METs, $\mathrm{mL} / \mathrm{kg} / \mathrm{dk}$ & $11,15 \pm 1,43$ & $9,27 \pm 2,15$ & $10,26 \pm 1,92$ & 0,013 & 0,201 \\
\hline
\end{tabular}

METs: equivalentes metabólicos; RM: ressonância magnética 
usando o protocolo Bruce. Todos os pacientes apresentaram capacidade aeróbica acima de 7 METs. Os testes de esforço foram encerrados a pedido dos próprios pacientes. Não foram observadas alterações significativas de segmento ST ( $\geq 1 \mathrm{~mm}$ ) ou de onda $\mathrm{T}$ em nenhum teste de esforço. Os valores do equivalente metabólico (METS) foram diferentes nos grupos controle, fluxo lento, e positivo à RMI $(11,15 \pm 1,43 ; 9,74 \pm$ 2,$05 ; 9,27 \pm 2,15$, respectivamente; $p=0,027$ para o grupo controle versus o grupo fluxo lento; $p=0,013$ para o grupo controle versus o grupo positivo à RM). Não houve diferenças de valores de MET entre o grupo controle e o grupo negativo à $\operatorname{RM}(11,15 \pm 1,43$ vs 9,74 $\pm 2,05 ; p=0,201)$ (Tabela 3 ).

\section{Discussão}

O principal achado deste estudo foi a detecção de tecido cicatricial à RMC em pacientes com fluxo lento na DAE. Os valores de NT-proBNP foram mais altos nos pacientes com fluxo lento e tecido cicatricial à RMC. Além disso, a capacidade aeróbica desses pacientes foi inferior quando comparada com a do grupo controle. Na literatura, os pacientes com fluxo coronário lento não haviam sido previamente avaliados por meio de RMC para detecção de presença de fibrose miocárdica. No nosso estudo, detectamos tecido cicatricial miocárdico na região da DAE em aproximadamente metade dos pacientes com o fenômeno do FCL na DAE. Esse achado foi estatística e clinicamente significativo quando comparado com o grupo controle. Entretanto, nós não avaliamos os pacientes com exames seriados de RMI ou medições seriadas de NT-proBNP. A ausência de tecido cicatricial nos pacientes restantes pode ser explicada pela limitação do nosso estudo. O desenvolvimento de tecido cicatricial no miocárdio requer a existência de um processo progressivo decorrente de danos contínuos durante anos. Assim sendo, a ausência de tecido cicatricial poderia ser o resultado do momento da avaliação. Como bem se sabe, o processo de formação de placa ateromatosa demora muitos anos, dependendo da presença de fatores de risco cardiovasculares, condições ambientais, fatores genéticos, e período de tempo. Os mesmos fatores podem também se aplicar ao fluxo coronário lento. Demonstramos com este estudo que o FLC não é de forma alguma inofensivo e que ele pode levar à cicatrização do tecido miocárdico ao final do respectivo processo patológico. O papel do NT-proBNP na fisiopatologia do FCL não está claro. Demonstrou-se que o peptídeo natriurético do tipo B é secretado dos cardiomiócitos em resposta à isquemia, e que essa secreção pode também ser independente do estresse da parede ventricular esquerda. ${ }^{17-20}$ Adicionalmente, além dos miócitos cardíacos, os fibroblastos podem secretar BNP e causar fibrose por indução das metaloproteinases de matriz através da liberação de BNP. ${ }^{21}$ No nosso estudo, os níveis de NT-proBNP não foram significativamente altos nos pacientes com fluxo lento. Entretanto, eles foram considerados altos nos pacientes com fluxo lento, nos quais as cicatrizes foram detectadas na RMC. Pode-se sugerir que os níveis de NT-proBNP foram elevados apenas na presença de fibrose suficiente em resposta ao fluxo coronário lento, que levou ao desenvolvimento de tecido cicatricial do miocárdio. A etiologia do $\mathrm{FCL}$ não foi claramente compreendida desde que foi descrita pela primeira vez. Embora o FCL possa ser uma consequência de alterações microvasculares, o aumento da resistência microvascular e a aterosclerose generalizada em estágio inicial também demonstraram desempenhar um papel na etiologia. 22,23 Além disso, tentou-se lançar mão das alterações histológicas e patológicas nas artérias coronárias para elucidar a etiologia. Em um estudo conduzido em pacientes com FCL, Mangieri et al., ${ }^{17}$ encontraram mudanças, tais como edema celular, hiperplasia fibromuscular, hipertrofia medial, proliferação miointimal, fibrose irregular, lesão capilar e redução do lúmen capilar, resultantes da biópsia do miocárdio, tendo alegado que essas alterações patológicas tornavam o fluxo sanguíneo mais lento através do aumento da resistência vascular. ${ }^{22,23}$ Ademais, no FCL, a ultrassonografia intravascular (USIV) revelou espessamento intimal difuso e calcificação generalizada, e a angigrafia coronária mostrou placas ateromatosas que não causam irregularidade luminal. ${ }^{24}$

Embora tenha-se relatada associação do fluxo coronário lento com muitas condições patológicas, ele parece desencadear uma doença aterosclerótica generalizada que é coincidente com uma doença microvascular na qual a disfunção endotelial encontra-se em primeiro plano. Podese considerer que a fibrose e a isquemia microvascular se desenvolvem no tecido miocárdico de pacientes com $\mathrm{FCL}$, como consequência das alterações que ocorrem no nível microvascular, e nosso estudo corrobora esse ponto de vista.

A função microvascular coronária deteriorada no $\mathrm{FCL}$ demonstrou uma relação com o aumento do risco de eventos cardiovasculares. ${ }^{25-27}$ Além disso, foi relatado que, nos pacientes com disfunção microvascular, o prognóstico é semelhante àquele observado na doença arterial coronariana obstrutiva, e que essa disfunção não é tão benigna quanto se pensava. ${ }^{28-30}$ As manifestações clínicas dessa patologia também estão associadas a achados significativos. Dor torácia atípica, ${ }^{16,31}$ dor torácia típica ${ }^{32}$ e dor torácica em repouso, que requerem intervenção urgente, ${ }^{4,33}$ frequentemente ocorrem em pacientes com fluxo coronário lento. Do mesmo modo, pacientes com FCL mostraram ser mais sintomáticos e suas internações hospitalares mais frequentes. ${ }^{34}$ Com base nisso, a RMC pode ser considerada uma boa escolha para investigar se o tecido miocárdico foi ou não afetado, além de oferecer uma opção favorável para avaliar a extensão da lesão, nos pacientes com FCL. A RMC de realce tardio possui alta resolução espacial. Com esse método, a fronteira entre o tecido infartado na parede do VE e o miocárdio viavél pode ser identificada através do exame da área do fluxo coronário lento. Além disso, a expansão transmural da área infartada pode ser determinada através desse método. Também é possível distinguir entre a isquemia vascular e não vascular em função da difusão do gadolínio.8 Na cardiomiopatia não isquêmica, o envolvimento do gadolínio é independente da perfusão vascular e ocorre na região subendocárdica. O envolvimento do gadolínio está diretamente associada à alimentação vascular na cardiomiopatia isquêmica. Além disso, esse envolvimento está localizado na região subendocárdica ou transmural. ${ }^{35}$

Panting et al.,31 demonstraram a hipoperfusão subendocárdica usando a RMC em pacientes com síndrome X, que acredita-se estar associada à disfunção microvascular. ${ }^{36} \mathrm{Do}$ mesmo modo, Lanza et al.,32 detectaram defeitos de perfusão 


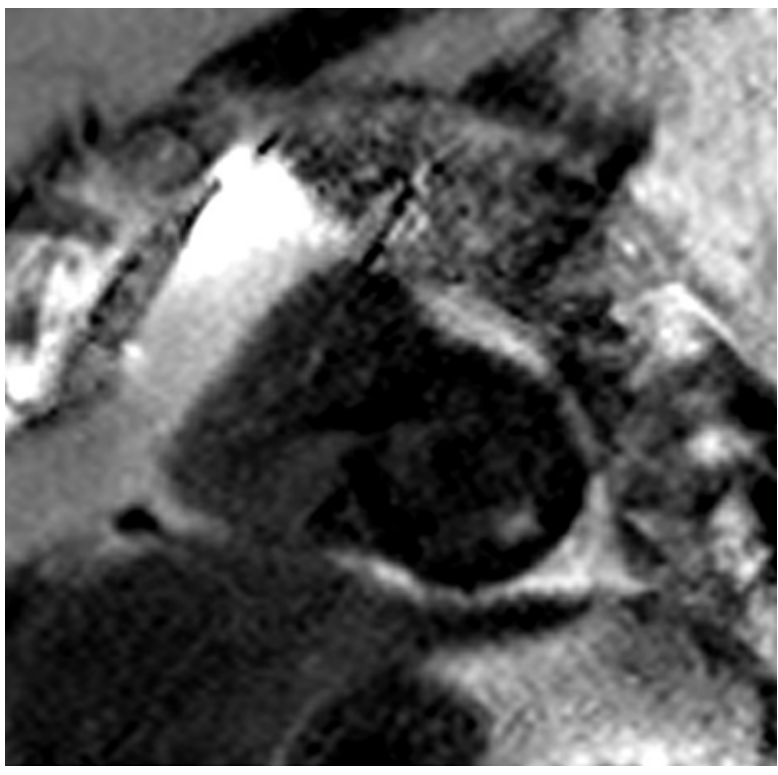

Figura 8 - Imagem de RMC com sequência PSIR pela técnica do contraste tardio no eixo curto, revelando áreas de realce focal nas regiões subepicárdica e subendocárdica localizadas nas paredes inferior e inferolateral do VE.

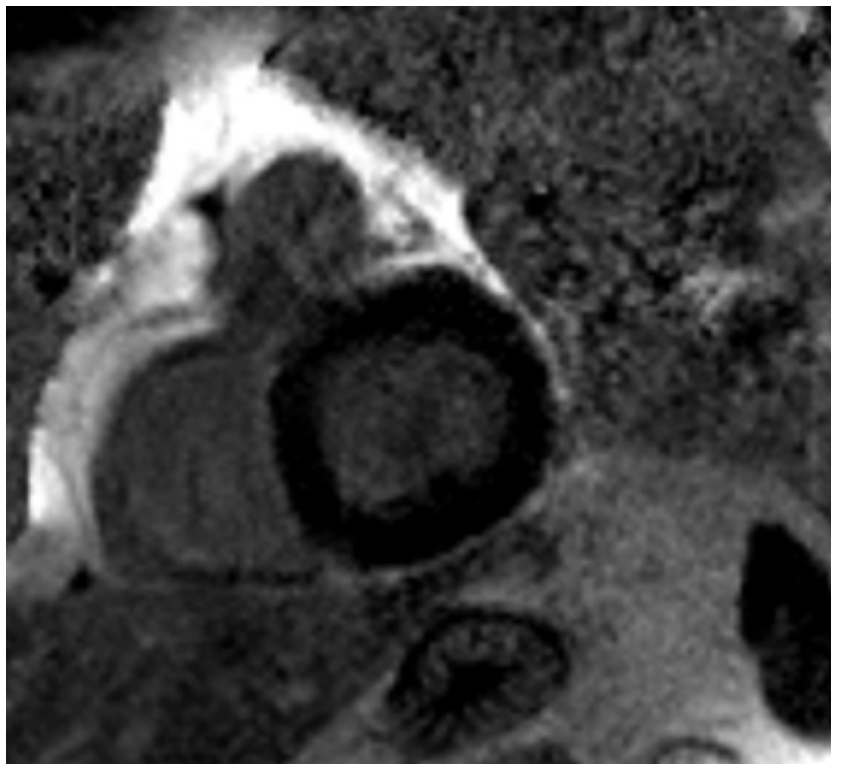

Figura 9 - Essa imagem mostra a aparência de um miocárdio normal. Essa imagem em particular foi adquirida na sequência PSIR. Não há realce anormal. 
na região da DAE do miocárdio nos pacientes com síndrome $X .{ }^{37}$ Demonstrou-se também que há uma importante relação entre a reserva de perfusão miocárdica, que é examinada através da RMC, e a disfunção microvascular coronariana, a qual é um precursor da aterosclerose precoce.$^{38}$

O NT-proBNP deve ser considerado após teste de esforço em pacientes com fluxo coronário lento. Ele pode fornecer informações sobre a fibrose cardíaca, embora ela possa ser afetada por diversas condições. Entretanto, não é possível realizar a RMC em todos os pacientes com contagem baixa de quadros TIMI devido à relação custo-benefício. A RMC pode ser considerada nos casos de pacientes com fluxo coronário lento em grau severo, dor torácica severa e valores elevados do biomarcador após o exercício. Devido ao pequeno número de pacientes do nosso estudo, não podemos fazer nenhuma recomendação em termos de tratamento, RMC ou controle do biomarcador. Todavia, este estudo poderá lançar luz sobre outros estudos, tanto no tocante ao tratamento (drogas antifibróticas) quanto aos exames (RMC, nível de NT-ProBNP, dentre outros).

\section{Limitações do Estudo}

Nosso estudo teve algumas limitações. Primeiramente, o número de pacientes foi pequeno. Em segundo lugar, as angiografias coronarianas foram realizadas por médicos diferentes e, embora as imagens angiográficas sejam padronizadas, houve diferenças pouco significativas entre as projeções. Finalmente, a técnica de ultrassom intravascular (USIV), capaz de mostrar a estrutura e as funções das artérias coronárias em detalhes, as medições da reserva de fluxo fracionada (RFF) e da pressão intracoronária (pressure wire), e os testes de acetilcolina não foram realizados no nosso estudo. Entretanto, a realização desses testes invasivos, com o seu potential de complicações, em pacientes sem estenose epicárdica não é apropriado por razões éticas.

\section{Referências}

1. Tatli E, Yildirim T, Aktoz M. Does coronary slow flow phenomenon lead to myocardial ischemia? Int J Cardiol. 2009;131(3):e101-2.

2. Kapoor A, Goel PK, Gupta S. Slow coronary flow--a cause for angina with ST segment elevation and normal coronary arteries. A case report. Int J Cardiol.. 1998;67(3):257-61.

3. Saya S, Hennebry TA, Lozano P, Lazzara R, Schechter E. Coronary slow flow phenomenon and risk for sudden cardiac death due to ventricular arrhythmias: a case report and review of literature. Clin Cardiol. 2008;31(8):352-5.

4. Beltrame JF, Limaye SB, Horowitz JD. The coronary slow flow phenomenon-a new coronary microvascular disorder. Cardiology. 2002;97(4):197-202.

5. Voelker W, Euchner U, Dittmann H, Karsch KR. Long-term clinical course of patients with angina and angiographically normal coronary arteries. Clin Cardiol. 1991;14(4):307-11.

6. Yurtdas M, Ozcan IT, Camsar A, Cicek D, Tamer L, Cin VG, et al. NT-Pro-BNP levels and their response to exercise in patients with slow coronary flow. Arq Bras Cardiol. 2012;99(6):1115-22.

7. Bruder O, Jensen C, Jochims M, Farazandeh M, Barkhausen J, Schlosser T, et al. Relation of B-type natriuretic peptide (BNP) and infarct size as assessed by contrast-enhanced MRI. Int J Cardiol. 2010;144(1):53-8.

\section{Conclusão}

Neste estudo, que foi conduzido para demonstrar o tecido cicatricial relacionado com o fenômeno do FLC, a RMC pela técnica do realce tardio apresentou resultados positivos. A RMC revelou tecido cicatricial nos pacientes com fluxo lento. Esses resultados sugerem que o fenômeno do fluxo lento pode acarretar alterações irreversíveis no tecido do miocárdio. As prováveis consequências dessas alterações devem ser investigadas em pesquisas futuras.

\section{Contribuição dos Autores}

Concepção e desenho da pesquisa: Candemir $M$, Şahinarslan A, Yazol M, Boyacı B; Obtenção de dados e análise e interpretação dos dados: Candemir M, Şahinarslan A, Yazol M, Öner YA, Boyacı B; Análise estatística e obtenção de financiamento: Candemir M, Boyacı B; Redação do manuscrito: Candemir M; Revisão crítica do manuscrito quanto ao conteúdo intelectual importante: Şahinarslan A, Öner YA.

\section{Potencial Conflito de Interesses}

Declaro não haver conflito de interesses pertinentes.

\section{Fontes de Financiamento}

O presente estudo foi financiado pelo Gazi University Scentific Research Projects.

\section{Vinculação Acadêmica}

Este artigo é parte de tese de Doutorado de Mustafa Candemir pela Gazi University.

\section{Aprovação Ética e Consentimento Informado}

Este estudo foi aprovado pelo Comitê de Ética do Gazi University sob o número de protocolo 83. Todos os procedimentos envolvidos nesse estudo estão de acordo com a Declaração de Helsinki de 1975, atualizada em 2013. O consentimento informado foi obtido de todos os participantes incluídos no estudo.
8. Kim RJ, Fieno DS, Parrish TB, Harris K, Chen EL, Simonetti O, et al. Relationship of MRI delayed contrast enhancement to irreversible injury, infarct age, and contractile function. Circulation. 1999;100(19):1992-2002.

9. Kim RJ, Wu E, Rafael A, Chen EL, Parker MA, Simonetti O, et al. The use of contrast-enhanced magnetic resonance imaging to identify reversible myocardial dysfunction. N Engl J Med. 2000;343(20):1445-53.

10. Ambale-Venkatesh B, Lima JA. Cardiac MRI: a central prognostic tool in myocardial fibrosis. Nat Rev Cardiol. 2015;12(1):18-29.

11. Saba L, Fellini F, De Filippo M. Diagnostic value of contrast-enhanced cardiac magnetic resonance in patients with acute coronary syndrome with normal coronary arteries. Jap J Radiol. 2015;33(7):410-7.

12. Lang RM, Badano LP, Mor-Avi V, Afilalo J, Armstrong A, Ernande L, et al. Recommendations for cardiac chamber quantification by echocardiography in adults: an update from the American Society of Echocardiography and the European Association of Cardiovascular Imaging. Eur Heart J Cardiovasc Imaging. 2015;16(3):233-70.

13. Gibbons RJ, Balady GJ, Bricker JT, Chaitman BR, Fletcher GF, Froelicher VF, et al. ACC/AHA 2002 guideline update for exercise testing: summary article: a report of the American College of Cardiology/American Heart Association Task Force on Practice Guidelines (Committee to Update the 1997 Exercise 
Testing Guidelines). Circulation. 2002;106(14):1883-92.

14. Cannon CP, McCabe CH, Diver DJ, Herson S, Greene RM, Shah PK, et al. Comparison of front-loaded recombinant tissue-type plasminogen activator, anistreplase and combination thrombolytic therapy for acute myocardial infarction: results of the Thrombolysis in Myocardial Infarction (TIMI) 4 trial. J Am Coll Cardiol. 1994;24(7):1602-10.

15. Gibson CM, Cannon CP, Daley WL, Dodge JT, Jr., Alexander B, Jr., Marble SJ, et al. TIMI frame count: a quantitative method of assessing coronary artery flow. Circulation. 1996;93(5):879-88.

16. Hawkins BM, Stavrakis S, Rousan TA, Abu-Fadel M, Schechter E. Coronary slow flow--prevalence and clinical correlations. Circulation .2012;76(4):936-42.

17. Mangieri E, Macchiarelli G, Ciavolella M, Barilla F, Avella A, Martinotti A, et al. Slow coronary flow: clinical and histopathological features in patients with otherwise normal epicardial coronary arteries. Cathet Cardiovasc Diagn. 1996;37(4):375-81.

18. Mosseri M, Yarom R, Gotsman MS, Hasin Y. Histologic evidence for smallvessel coronary artery disease in patients with angina pectoris and patent large coronary arteries. Circulation. 1986;74(5):964-72.

19. Cin VG, Pekdemir H, Camsar A, Cicek D, Akkus MN, Parmaksyz T, et al Diffuse intimal thickening of coronary arteries in slow coronary flow. Jap Heart J. 2003;44(6):907-19.

20. Schachinger V, Britten MB, Zeiher AM. Prognostic impact of coronary vasodilator dysfunction on adverse long-term outcome of coronary heart disease. Circulation. 2000;101(16):1899-906.

21. Suwaidi JA, Hamasaki S, Higano ST, Nishimura RA, Holmes DR, Jr., Lerman A. Long-term follow-up of patients with mild coronary artery disease and endothelial dysfunction. Circulation. 2000;101(9):948-54.

22. Halcox JP, Schenke WH, Zalos G, Mincemoyer R, Prasad A, Waclawiw $\mathrm{MA}$, et al. Prognostic value of coronary vascular endothelial dysfunction. Circulation. 2002;106(6):653-8.

23. Delcour KS, Khaja A, Chockalingam A, Kuppuswamy S, Dresser T. Outcomes in patients with abnormal myocardial perfusion imaging and normal coronary angiogram. Angiology. 2009;60(3):318-21.

24. Bugiardini R, Manfrini O, Pizzi C, Fontana F, Morgagni G. Endothelial function predicts future development of coronary artery disease: a study of women with chest pain and normal coronary angiograms. Circulation. 2004;109(21):2518-23.

25. Bugiardini R, Bairey Merz CN. Angina with "normal" coronary arteries: a changing philosophy.JAMA. 2005;293(4):477-84.
26. Sadr-Ameli MA, Saedi S, Saedi T, Madani M, Esmaeili M, Ghardoost B. Coronary slow flow: Benign or ominous? Anatol J Cardiol. 2015;15(7):531-5.

27. Amirzadegan A, Motamed A, Davarpasand T, Shahrzad M, Lotfi-Tokaldany M. Clinical characteristics and mid-term outcome of patients with slow coronary flow. Acta Cardiol. 2012;67(5):583-7.

28. Gokce M, Kaplan S, Tekelioglu Y, Erdogan T, Kucukosmanoglu M. Platelet function disorder in patients with coronary slow flow. Clin Cardiol. 2005;28(3):145-8.

29. Yilmaz H, Demir I, Uyar Z. Clinical and coronary angiographic characteristics of patients with coronary slow flow. Acta Cardiol.2008;63(5):579-84.

30. Hoey ET, Gulati GS, Ganeshan A, Watkin RW, Simpson H, Sharma S. Cardiovascular MRI for assessment of infectious and inflammatory conditions of the heart. AJR Am J Roent. 2011;197(1):103-12.

31. Panting JR, Gatehouse PD, Yang GZ, Grothues F, Firmin DN, Collins $P$, et al. Abnormal subendocardial perfusion in cardiac syndrome $X$ detected by cardiovascular magnetic resonance imaging. N Engl J Med. 2002;346(25):1948-53

32. Lanza GA, Buffon A, Sestito A, Natale L, Sgueglia GA, Galiuto L, et al. Relation between stress-induced myocardial perfusion defects on cardiovascular magnetic resonance and coronary microvascular dysfunction in patients with cardiac syndrome X. J Am Coll Cardiol. 2008;51(4):466-72.

33. Wohrle J, Nusser T, Merkle N, Kestler HA, Grebe OC, Marx N, et al. Myocardial perfusion reserve in cardiovascular magnetic resonance: Correlation to coronary microvascular dysfunction. J Cardiovasc Magn Res. 2006;8(6):781-7.

34. Goetze JP, Gore A, Moller CH, Steinbruchel DA, Rehfeld JF, Nielsen LB. Acute myocardial hypoxia increases BNP gene expression. FASEB J. 2004;18(15):1928-30.

35. Goetze JP, Christoffersen C, Perko M, Arendrup H, Rehfeld JF, Kastrup J, et al. Increased cardiac BNP expression associated with myocardial ischemia. FASEB J. 2003;17(9):1105-7.

36. Bibbins-Domingo K, Ansari M, Schiller NB, Massie B, Whooley MA. B-type natriuretic peptide and ischemia in patients with stable coronary disease: data from the Heart and Soul study. Circulation. 2003;108(24):2987-92.

37. Hama N, Itoh H, Shirakami G, Nakagawa O, Suga S, Ogawa Y, et al. Rapid ventricular induction of brain natriuretic peptide gene expression in experimental acute myocardial infarction. Circulation. 1995;92(6):1558-64.

38. Tsuruda T, Boerrigter G, Huntley BK, Noser JA, Cataliotti A, CostelloBoerrigter LC, et al. Brain natriuretic Peptide is produced in cardiac fibroblasts and induces matrix metalloproteinases. Circ Res. 2002;91(12):1127-34. 